\title{
Development of a Service Robot System for a Remote Child Monitoring Platform
}

\author{
Taewoo $\mathrm{Han}^{1}$ and Yong-Ho $\mathrm{Seo}^{2, *}$ \\ ${ }^{1}$ Department of Game and Multimedia, Woosong University \\ 171 Dongdaejeon-ro, Dong-gu, Daejeon, Republic of Korea \\ ${ }^{2}$ Department of Intelligent Robot Engineering, Mokwon University \\ 88 Doanbuk-ro, Seo-gu, Daejeon, Republic of Korea \\ bluebird@wsu.ac.kr,yhseo@mokwon.ac.kr
}

\begin{abstract}
Network-based intelligent surveillance is becoming more popular, and remote monitoring platforms using web cameras with mobile robots can be used as child monitoring platforms in infant caring facilities. This paper presents a prototype of a service robot system as a nextgeneration intelligent surveillance system for child monitoring. Im order to achieve this, a cooperative server-client control scheme is developed that has multiple users and a remote robot that utilizes an appropriate communcation method. The experimental results demonstrate the feasibility of the proposed service robot kystem through integrating the required skills into the prototype of an infant caring sureillance system.
\end{abstract}

Keywords: Intelligent Surveillance, Service Robot Platform, Remote Child Monitoring, Robot Control Right Transfer, Cooperdive Server-Client Control

\section{Introduction}

Recently, the evolution of the concept of natural surveillance using robots has emerged from the concept of surveillance asing fixed closed circuit television (CCTV). Therefore, monitoring services that can satisfy teachers, children, and parents need to be built for general use in childcare facilities [1]. Recently, child abuse has emerged as a social issue in the home and at childcare centers, both domestically and internationally; as a result, mandatory static CCTV installations are inereasingly commonplace in daycare centers and kindergartens. However, statically installed cameras have blind spots, so there is a significant need for a different solution to avoid these blind spots.

Mandatory CCTV is currently used in many daycare centers and kindergartens, but some surveys have indicated that CCTV installation infringes on teachers' dignity and human rights, so an appropriate compromise must be sought.

Furthermore, the display features of the robots that have been installed in daycare centers are emphasized so people think they are moving computers. Also, teachers tend to be reluclant to use the robots due to a fear of damaging them. Therefore, an alternative solution is required.

In this paper, a number of service robots were placed in several spaces and servers were built for multiple users to remotely monitor each robot. Furthermore, the technology required in order to have more than one robot connected to a wireless access point (AP) and the server was developed. Through developing application clients for remote monitoring and service

${ }^{*}$ Corresponding author. 
robot control, it is demonstrated that the application proposed through the technology of remote control and monitoring using smartphones can be applied as an interface that can control the service robot.

\section{Remote Monitoring Service Robot System}

Through placing a number of service robots in several spaces and through building a server that can monitor each robot in multiple remote locations, a system in which more than one mobile robot (camera, microphone, speaker mounted-type) is placed in at least three spaces (nursery facilities) is designed; more than one mobile robot has access to the wireless AP (Wi-Fi) and is connected to the server. The system is constructed through securing the server for multiple remote monitoring capabilities with fixed IPs in a data center.

The server is accessed using Android-based [2] application client technology. Then after accessing the server, the space (e.g. nursery facilities) to be monitored and the remote monitor service robots present in the nursery facilities are selected, and real-time con rol services can be implemented between the service robots and application client based on the control interface system server. The server is constructed with many obots, smartphone multiple application client accesses, and massive data processing through interlocking more than three application clients and more than one robot placed in at least three spaces (nursery facilities) in the same manner as the Windows operating system-based method. The overall system is summarized in Figure 1.

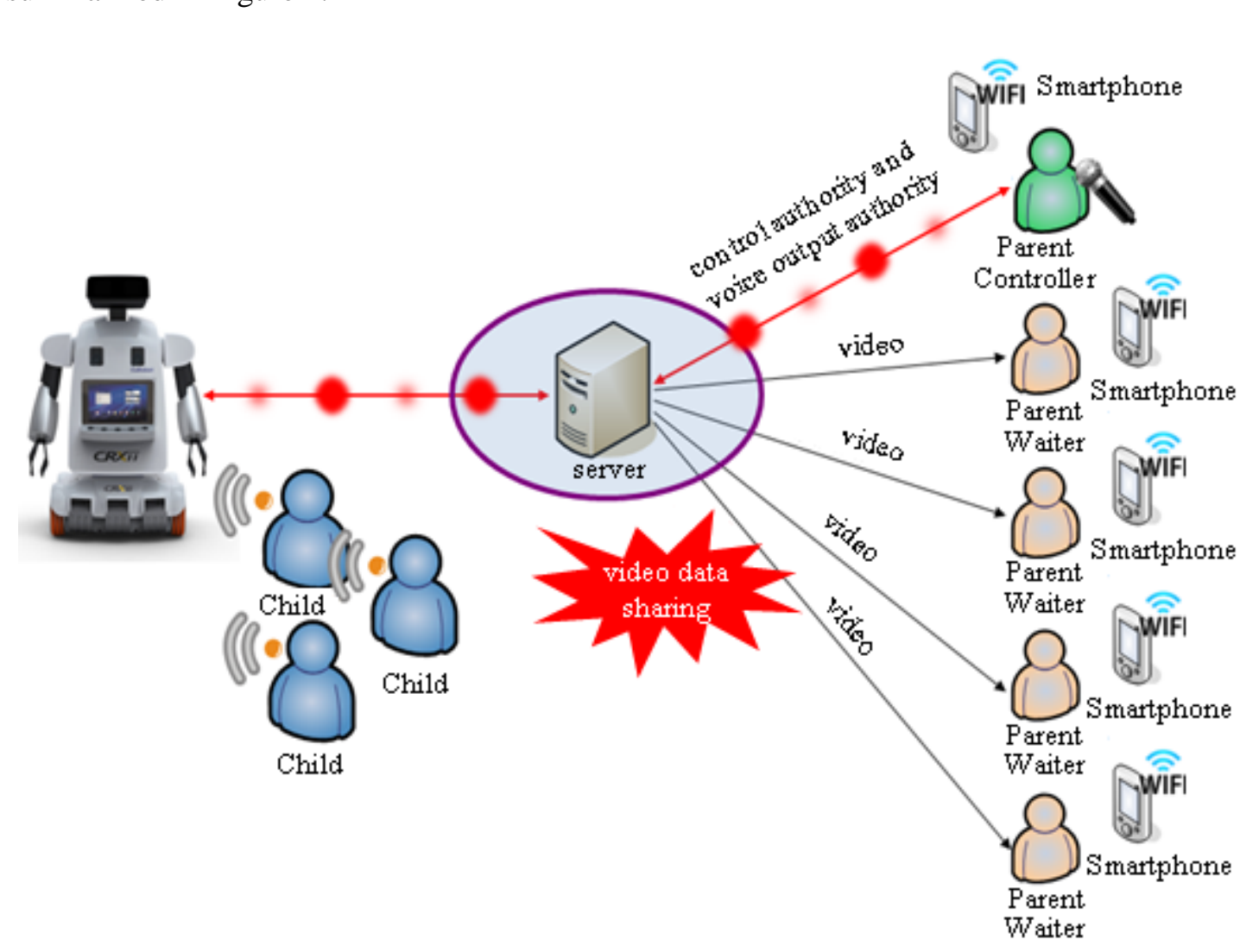

Figure 1. Overview of the Remote Monitoring Service Robot System 
The service robot platform used in this research was developed under a joint development research program between Mokwon University and CNRobot Inc. [3]. The hardware configuration and an internal view are presented in Figure 2, while Figure 3 illustrates the robot appearance. The mobile robot platform has an embedded camera pan/tilt; it moves using two wheels; and the camera video data is sent to the server using the embedded WiFi module and HRI using the microphone and speaker.
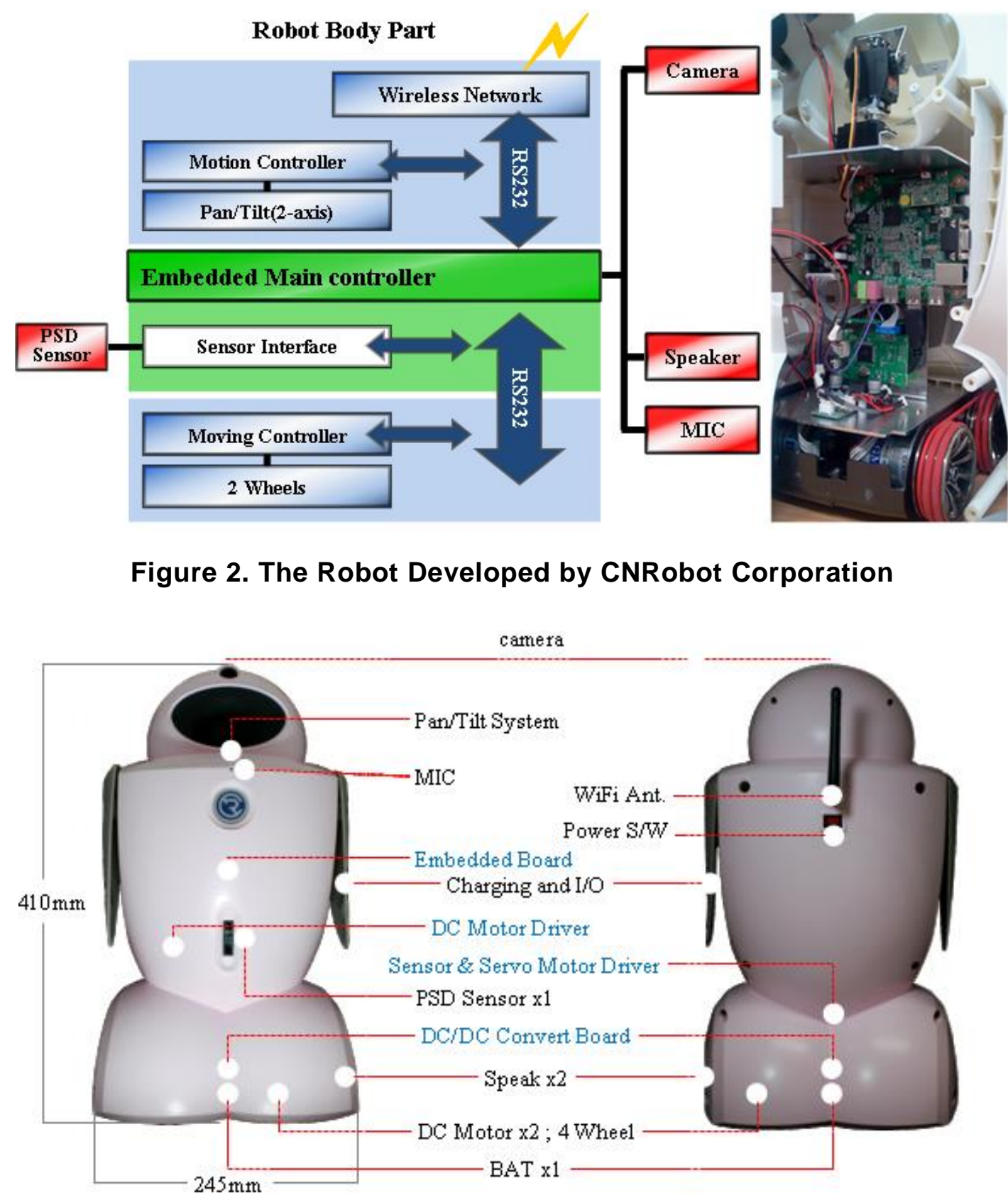

Figure 3. Appearance of the Robot Developed by CNRobot Corporation 
The system used to control the service robot is presented in Figure 4. Using the network integration system structure design, the fusion method analysis is implemented remotely. Furthermore, the basic design of the video/audio monitoring and control algorithm structure, the multiple application client smart devices, and the efficient network integration system framework design for the robots, server, and smart devices are designed using OPRoS feature analyses and RTC middleware [4-5].

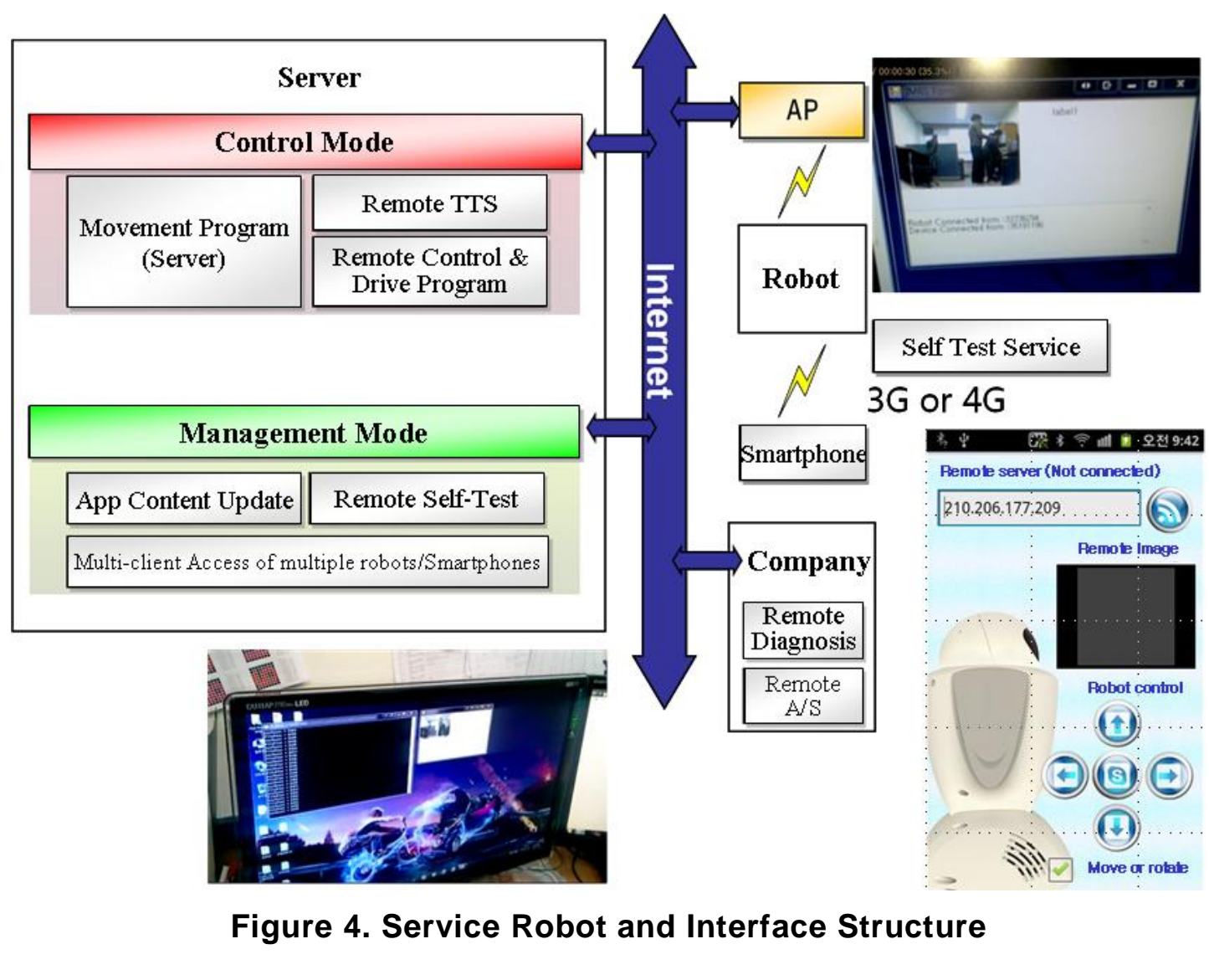

\section{Method of Transfering Robot Platform Control Rights}

This method enables the control of the robots, which can be remotely accessed by parents, and includes the management server with the remote connections when offsite parents access the server ûsing their smart devices in order to take control of the robots and observe the situation. In also provides server access for a number of users through their smart devices in the remote control standby mode of the robots [6].

The connected users can obtain the video and audio from the classroom situation using the smart device application; even users who do not have robot control rights can watch video and audio that is obtained through the robot if they access the server. In a control standby situation where no one has robot control rights, if one user applies for robot control rights, it is assigned to the user. Then, the remaining time until the robot control rights can be transferred is displayed in the application of the other remote connections. If another remote connection that is on the control rights waiting list applies for control rights transfer using the smart device application, the remaining time is displayed to the applicant. The remaining time 
of the current control rights and use times of the applicant who applied are also displayed to the other connections. Refer to Figure 5.

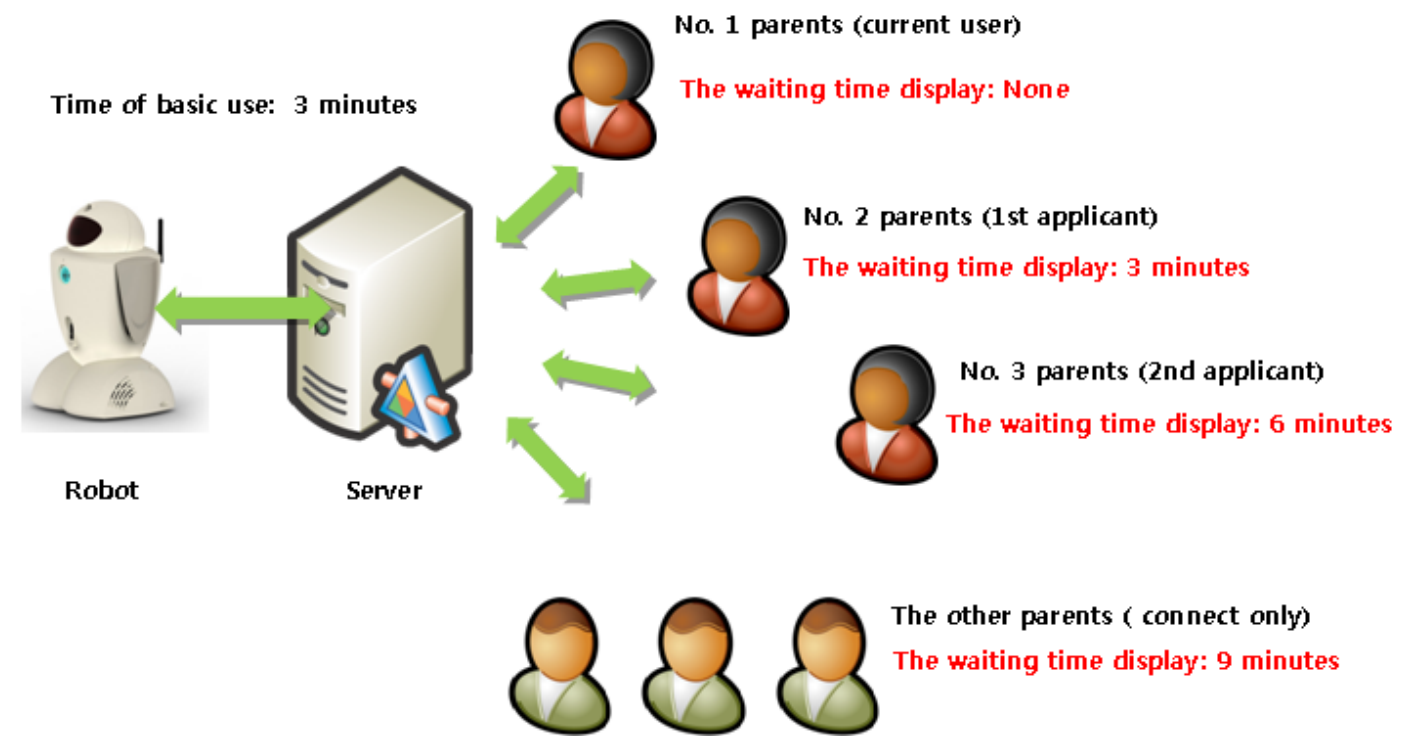

Figure 5. The Control Rights Transfer of the Teacher is Not Applied

In addition to the users using the application, kindergarten teachers receive the control rights transfer as the first priority when they apply for the control rights transfer regardless of the remaining waiting time. In this situation, the waiting time and use time of the kindergarten teacher are displayed to remaining connections and wait list. The alarm sounds for the waiting user 30 seconds before the waiting time finishes in order to alert the waiting user to the transfer order. The waiter who will recelve the control rights next will gain control through clicking the robot contron rights transfer button; if the transfer button is not clicked within 30 seconds of the transfen time, the transfer right moves to the next user. The waiting time is displayed in real time to the waiting users and connectors. If there is a change in the transfer waiting users, changes in the waiting time are notified through push notifications. Refer to Figure 6.

In summary, in the process of transferring the robot control rights for remote robot control and communication transfer waiting times, and alarms are displayed differently according to the control applicants and access waiters. Therefore, the inconvenience can be reduced in the waiting process and the service use time can be predicted in advance. A flow chart of the control rights transfer is presented in Figure 7.

\section{Experiment}

The server was built in a general Windows-based operating system environment and then the data communication was tested using a $\mathrm{C \#}$ application client program [7]. The program could undertake functions such as connect with the robot, display the robot and server status, display the robot video, and control the robot, as shown in Figure 8. 


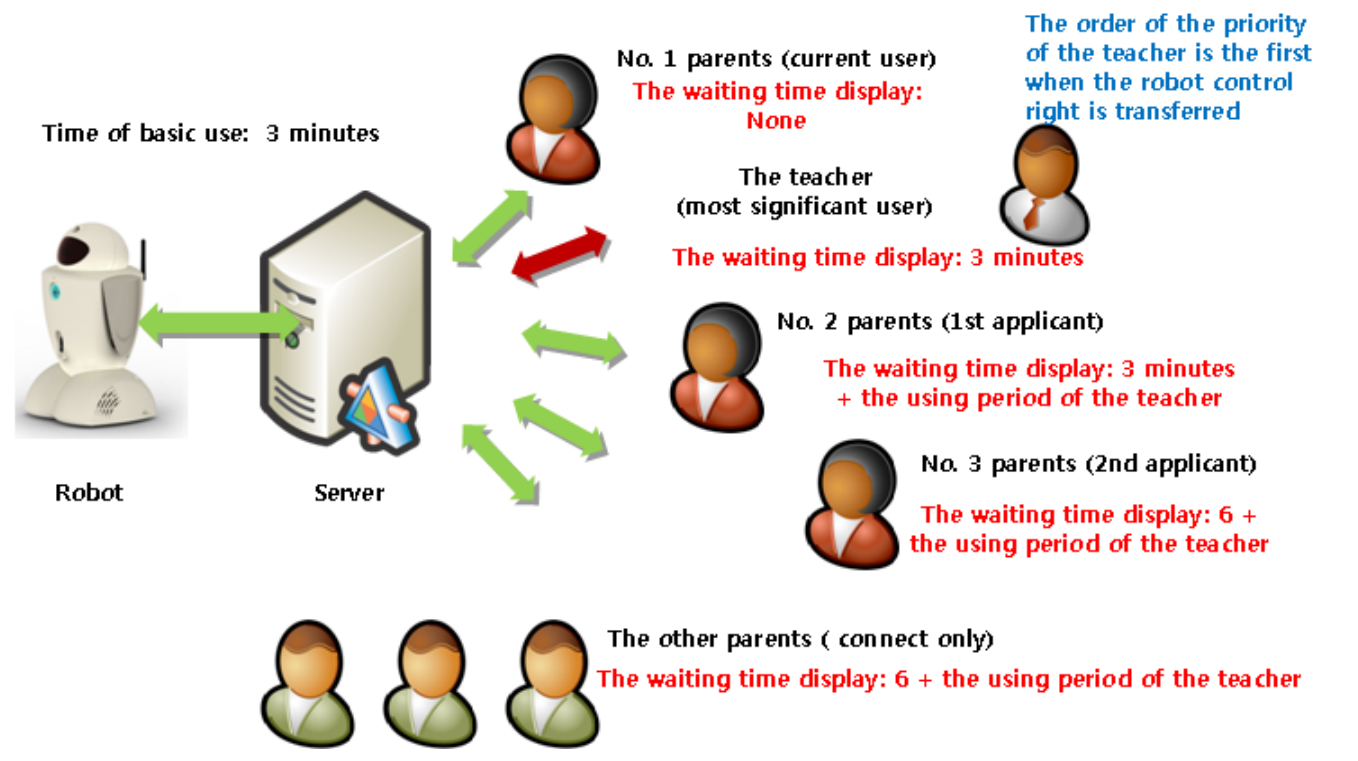

Figure 6. The Control Rights Trans er of the Teacher is Applied

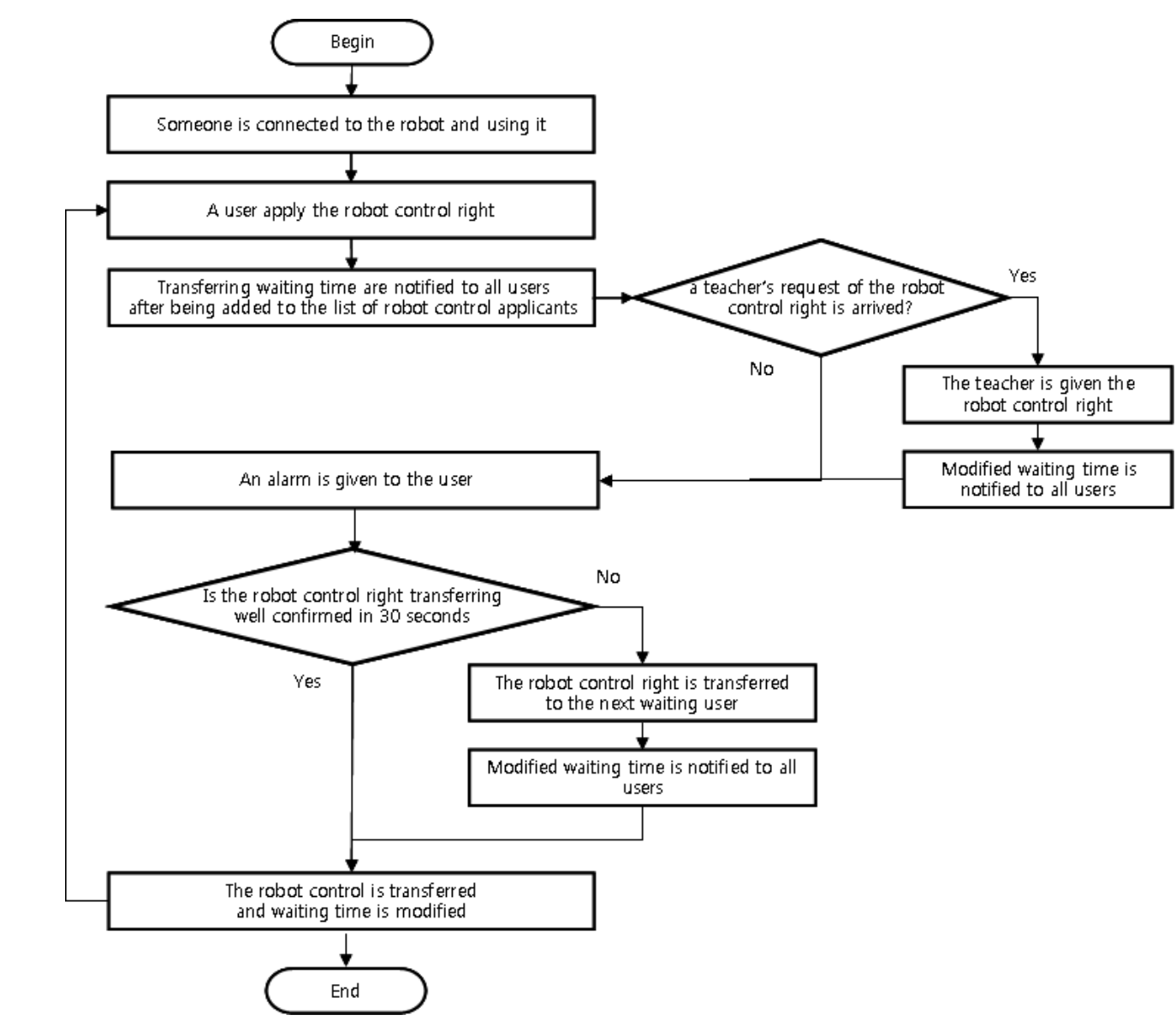

Figure 7. Flow Chart for the Control Rights Transfer 


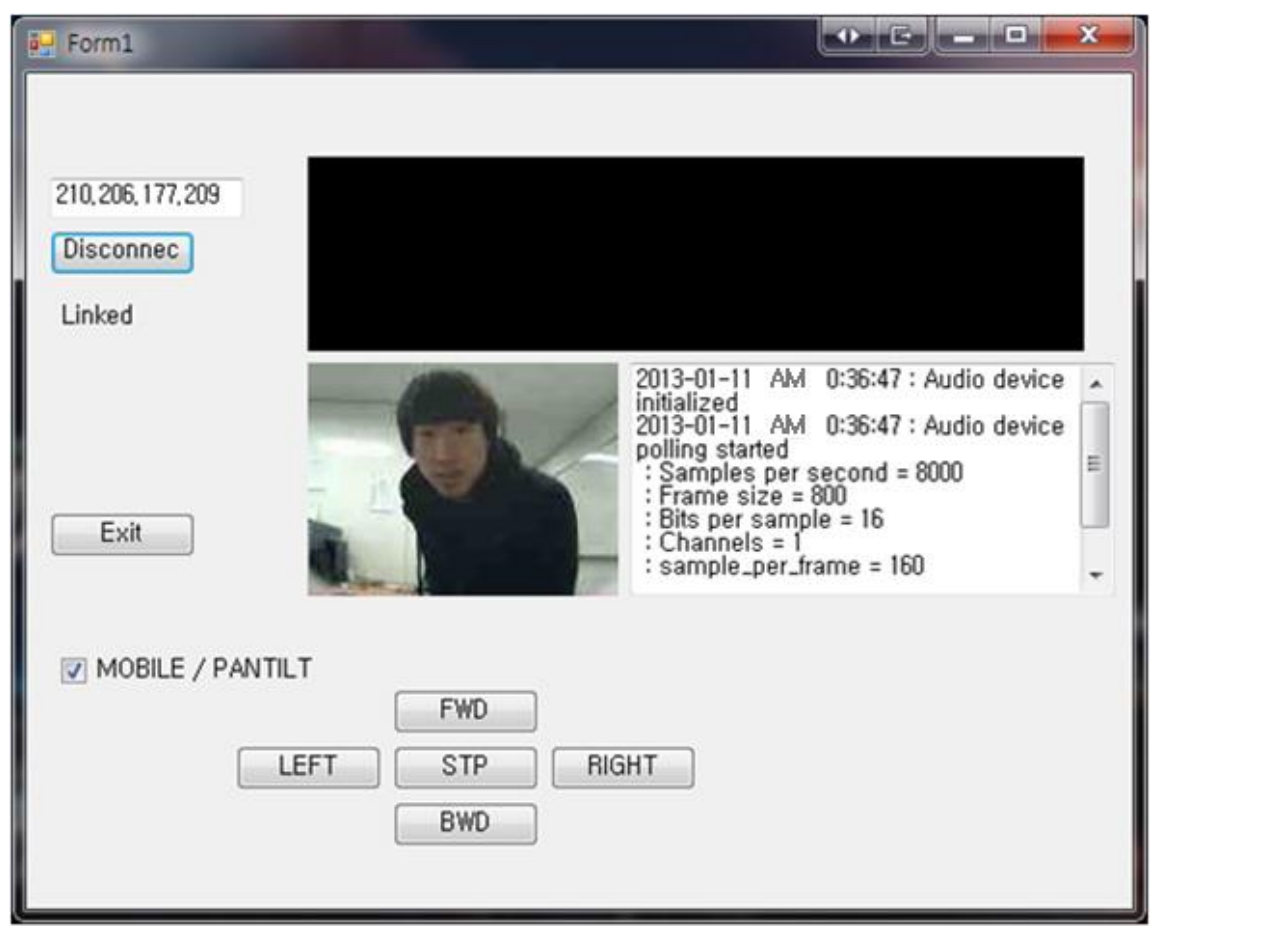

Figure 8. Output Screen on PC Client

Figure 9 presents the robot image data and robotsensor data identified in the server.

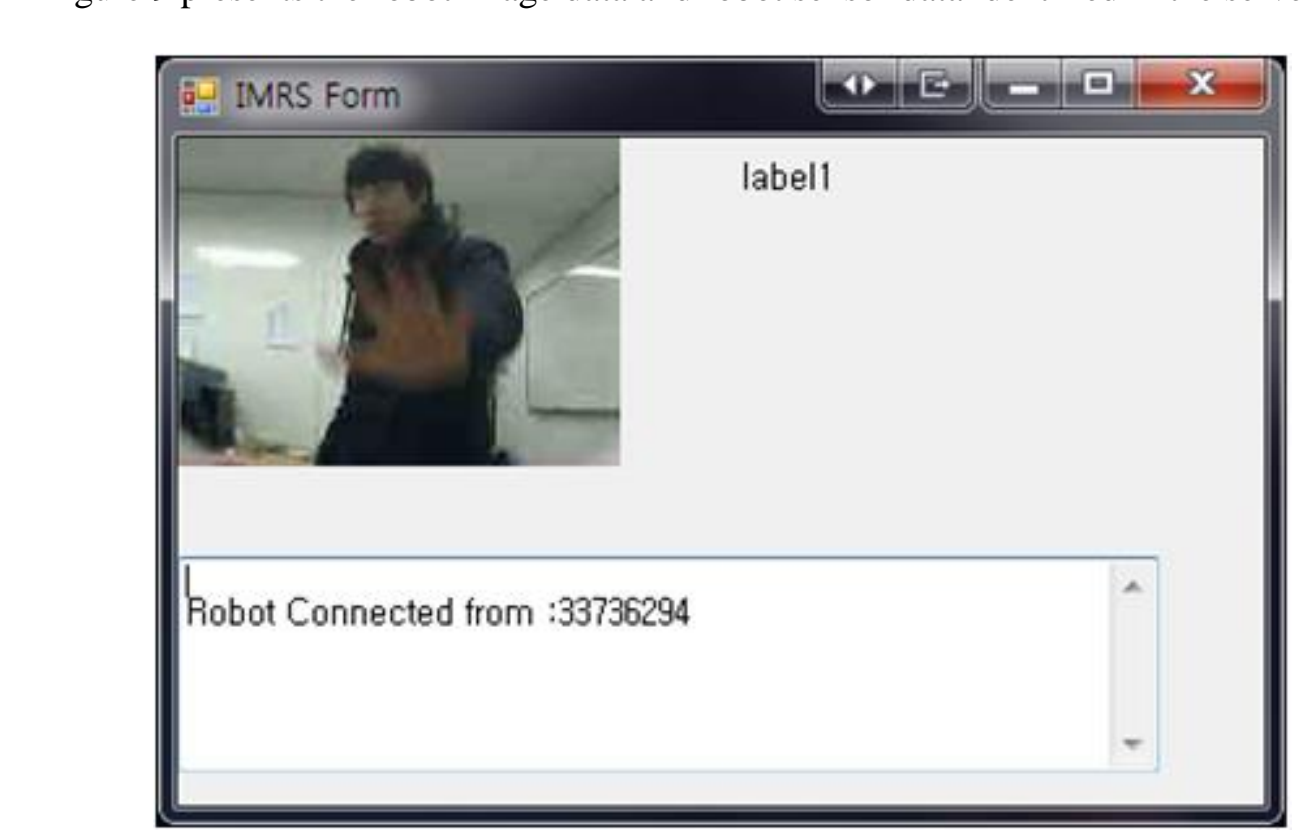

Figure 9. Output Screen on PC Server

The server can conduct management functions such as connection with the robot and the clients, display of the robot and server status, transfer of images and voice data from robots to the specified clients, and transfer of control messages to the robots. The demonstration of the robot motion through server linkage is shown in Figure 10. 


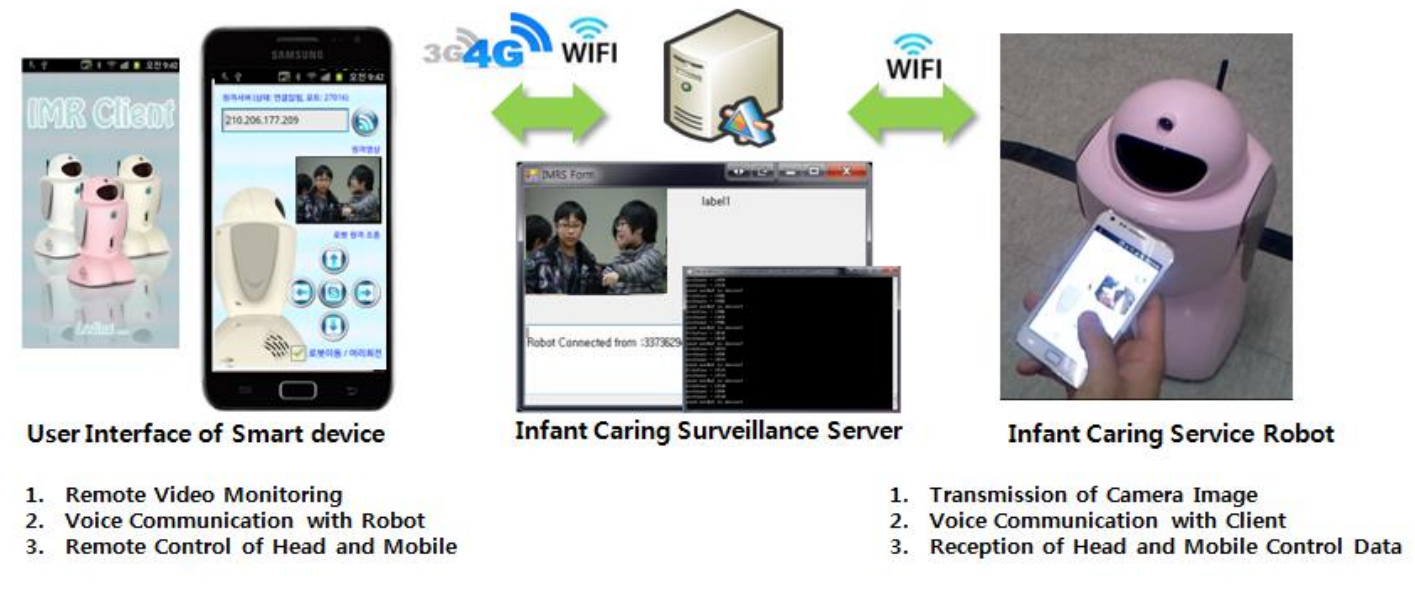

Figure 10. Demonstration of the Robot Motion through Server Linkage

\section{Conclusion}

The server was designed for multiple users to remitely monitor the robot and an application client was developed for the remote monitormg and service robot control. Furthermore, a user interface was developed to enable smart devices to connect and monitor the situation using the service robot. Moreover, the technology that enables the obtaining and displaying of the mobile robot remote monitoring of video/audio in the selected space was developed. The robot access technology was established through selecting the facilities to be monitored after accessing the server through the application client. The results of this research are provided in Table 1.

Table 1. Results of the Applidation Development for the Service Robot

\begin{tabular}{|c|c|}
\hline Classification & Development Results \\
\hline Speed and runt/me & Speed: $0.8 \mathrm{~m} / \mathrm{s}$, Runtime: $6 \mathrm{hrs}$ \\
\hline Head movement & Pan: $180^{\circ}$, tilt: $90^{\circ}$ \\
\hline Range detection & PSD: $80 \mathrm{~cm}$ under \\
\hline \multirow{2}{*}{$\begin{array}{l}\text { Remoteldata } \\
\text { transmission with } \\
\text { control }\end{array}$} & $\begin{array}{l}\text { Remote audio input and output and transmission, remote robot } \\
\text { control }\end{array}$ \\
\hline & $\begin{array}{l}\text { Development of a mobile app that can control the overall operation } \\
\text { of the robot }\end{array}$ \\
\hline Alerts for robot status & Alerts for low battery, wheel jamming, obstacle detection \\
\hline Robot control method & Robot control through server \\
\hline $\begin{array}{l}\text { Maximum number of } \\
\text { simultaneous access }\end{array}$ & $\begin{array}{l}\text { Simultaneous access of up to three robots and ten smartphones and } \\
\text { video/audio data processing }\end{array}$ \\
\hline
\end{tabular}




\section{References}

[1] Y. Seo, "Remote Control and Monitoring of an Omni-directional Mobile Robot with a Smart Device", 5th International Conference on Convergence and Hybrid Information Technology, ICHIT 2011, Daejeon, Korea, September 22-24, pp. 286-294, 2011.

[2] Android developers, http://developer.android.com/guide/basics/what-is-android.html.

[3] http://cnrobot.co.kr.

[4] Taewoo Han and Yong-Ho Seo, "Mixed Reality System for Virtual Interior Design", International Journal of Smart Home, Vol. 7, No.3, pp. 133-142, May 2013.

[5] Young Joon Kim and Yong-Ho Seo, "Simple Programming Language for Creating a Simulation Environment with Mobile Robots", International Journal of Smart Home, Vol. 7, No.4, pp. 77-84, July 2013.

[6] David Ramada, Carlos Domínguez, Houcine Hassan, Alfons Crespo, "Flexible Real-time Embedded Architecture for Advanced Service Robots, ”, International Journal of Smart Home, Vol. 2, No.4, pp. 59-74, October 2008.

[7] Microsoft .NET Framework Developer Center, http://msdn.microsoft.com/netframework.

\section{Authors}

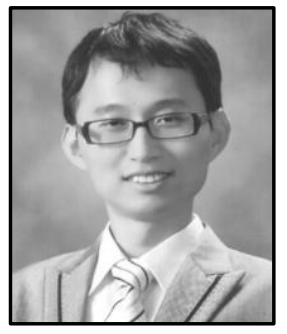

Taewoo Han, he received his BS. and M.S. dêgrees in the Department of Computer Science and his Ph.D. degree in the Department of Electrical Engineering and Computer Science from KAIST (Korea Advanced Institute of Science and Technology) in 1996, 1998, and 2005 respectively. He was an intern researchecat the institute of information and electronics, KAIST Since March 2007, he has been currently a Professor and a Chair of the Department of Game and Multimedia, Woosong University. Als research interests include mixed reality, vision system, computer graphics, ubiquitous computing and multimedia.

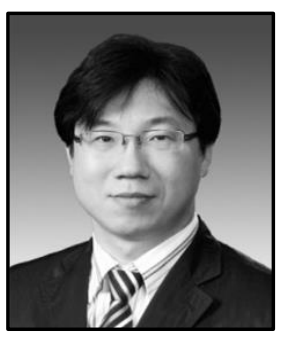

Yong-Ho Seo, the received his BS and MS degrees from the Department of Electrical Engineering and Computer Science, KAIST, in 1999 and 2001, respectively. He also received a $\mathrm{PhD}$ degree at the Artificial Intelligence and Media Laboratory, KAIST, in 2007. He was an Intern Researcher at the Robotics Group, Microsoft Research, Redmond, WA in 2007. He was a consultant at Qualcomm CDMA Technologies, San Diego, CA in 2008. He is currently a Professor and a Chair of the Department of Intelligent Robot Engineering, Mokwon University. His research interests include humanoid robot, human-robot interaction, robot vision and wearable computing.

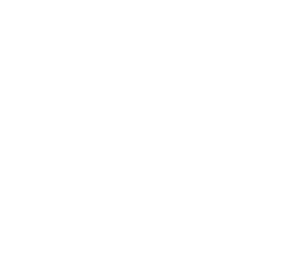


International Journal of Smart Home

Vol.8, No.5 (2014)

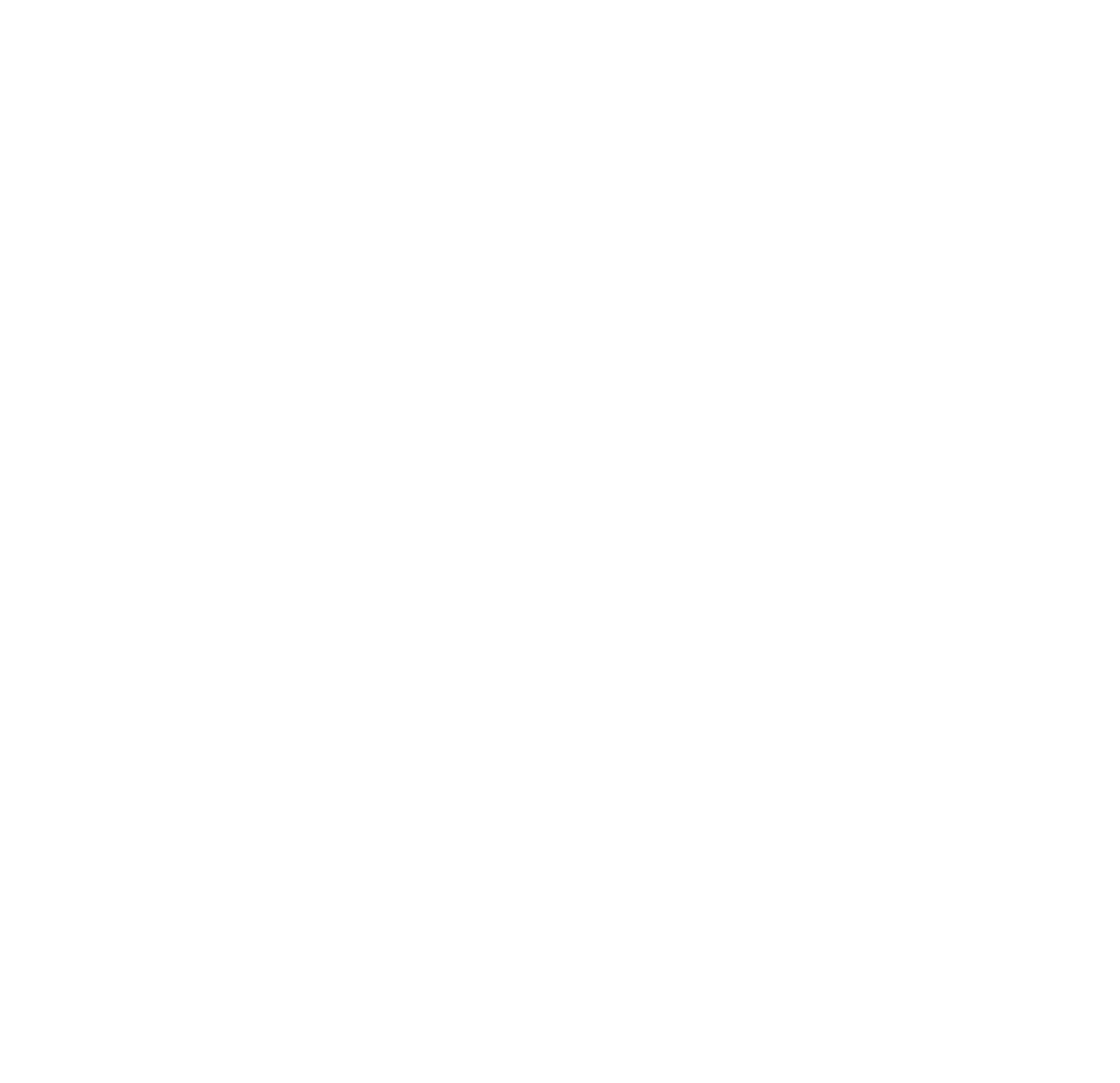

